Article

\title{
Broadband Multichannel Optical Vortex Generators via Patterned Double-Layer Reverse-Twist Liquid Crystal Polymer
}

\author{
Hanqing Zhang ${ }^{1}$, Wei Duan ${ }^{1,2, *}$, Ting Wei ${ }^{1}$, Chunting $\mathrm{Xu}^{1}$ and Wei $\mathrm{Hu}{ }^{1, *} \mathbb{C}$ \\ 1 National Laboratory of Solid State Microstructures, College of Engineering and Applied Sciences, \\ Nanjing University, Nanjing 210093, China; mf1834073@smail.nju.edu.cn (H.Z.); \\ weiting.nju@outlook.com (T.W.); xuchunting.nju@foxmail.com (C.X.) \\ 2 School of Instrumentation and Optoelectronic Engineering, Beihang University, Beijing 100191, China \\ * Correspondence: wduan@buaa.edu.cn (W.D.); huwei@nju.edu.cn (W.H.); \\ Tel.: +86-25-83597400 (W.D \& W.H.)
}

Received: 31 August 2020; Accepted: 27 September 2020; Published: 29 September 2020

\begin{abstract}
The capacity of an optical communication system can be greatly increased by using separate orbital angular momentum (OAM) modes as independent channels for signal transmission and encryption. At present, a transmissive OAM mode generator compatible with wavelength division multiplexing is being highly pursued. Here, we introduce a specific double-layer reverse-twist configuration into liquid crystal polymer (LCP) to overcome wavelength dependency. With this design, broadband-applicable OAM array generators are proposed and demonstrated. A Damman vortex grating and a Damman q-plate were encoded via photopatterning two subsequent LCP layers adopted with oppositely handed chiral dopants. Rectangular and hexagonal OAM arrays with mode conversion efficiencies exceeding $40.1 \%$ and $51.0 \%$ in the ranges of 530 to $930 \mathrm{~nm}$, respectively, are presented. This provides a simple and broadband efficient strategy for beam shaping.
\end{abstract}

Keywords: beam shaping; liquid crystals; orbital angular momentum; geometric phase

\section{Introduction}

The optical vortex (OV), an optical field featured by a helical phase front [1], has attracted extensive attention over the past few decades. The phase factor of an OV $\mathrm{e}^{i m \varphi}$ is proportional to the azimuth $\varphi$, where $m$ is the topological charge. The uncertainty of the phase front in the center of the OV makes a phase singularity, leading to a donut-like intensity distribution [2]. The Poynting vector of the beam twists $m$ times around its propagation axis in a single wavelength, and the spinning makes the beam carry orbital angular momentum (OAM), which is quantitively depicted by $m$. Due to the infinity of $m$, the OAM forms a Hilbert space with infinite dimensions and adds a new degree of freedom for the manipulation of light [2]. An OV can be generated by a liquid crystal device called a q-plate [3] where $\mathbf{q}=m / 2$ and shows great potential in non-contact manipulation [4], quantum computing $[5,6]$, and information encoding and storage [7]. Especially, with different OAM modes as independent channels for signal transmission or encryption, the transmission capacity of optical communication can be dramatically extended [8,9] via mode-division multiplexing [10]. OV mode conversion efficiency is highly dependent on wavelength, thus hindering its compatibility with present wavelength-division multiplexing techniques. Methods for highly-efficient and parallel OV processing in a broadband remain highly pursued.

Many efforts have been made to tackle this challenge. Damman vortex gratings (DVGs) [11] were introduced to generate OV arrays with equal energy distribution among multiple diffraction orders that carry different OAM states. Though the uniformity among various OAM states has been 
well-addressed, these DVGs still suffer from significant wavelength-dependent efficiency. To solve this problem, the phase retardation/shift of OV array generators should be continuously and precisely tuned. Liquid crystals (LCs) are excellent optical anisotropic materials for phase modulations due to their large birefringence and external field tunability. Previously designed LC-mediated DVGs [12] allowed for tunable optimized bands for OV generation via the electrical alternation of the output phase retardations. More recently, the geometric phase was discovered for the light reflected off planar cholesteric LC chiral superstructures. For such a device, the reflective efficiency is constant in the Bragg band and the phase can be freely manipulated via initial alignment control [13-16]. A Damman-encoded vortex grating was presented with a wide photonic band gap from green to red. In addition, such grating can transform a Gaussian beam to a series of reflective wave carrying different OAMs in different orders with very good energy uniformity in a broadband to $116 \mathrm{~nm}$ [17]. Until now, the transmissive elements suitable for generating and separating of multiple OVs remained formidable.

In this paper, we introduce a double-layer reverse-twist configuration to compensate for the dispersion over a large wavelength range. By further encoding a DVG phase profile into a photopatterned liquid crystal polymer (LCP), multichannel OV arrays were generated equally-efficiently. Thanks to the broadband spin-orbit interaction, uniform OV arrays were achieved in equally-high-efficiency across visible and part of near-infrared range. This work may provide a solution for broadband and arbitrary beam shaping in a transmissive way.

\section{Materials and Methods}

\subsection{Design and Principle}

Damman grating (DG) is a typical binary optics that provides equal energy distribution among all desired diffraction orders, providing a promising strategy for parallel beam processing [18,19]. Similar to DG, a DVG is a binary hologram calculated from the interference between a tilt plane wave and an OV. Figure 1 a exhibits the phase pattern of a $2 \times 2$ DVG, which is a two-dimension (2D) DVG integrated by two one-dimension (1D) DVG with different $m$ in $x$ and $y$ directions. The phase distribution $\varphi$ of a 1D DVG (grating vector along $x$ for instance) is denoted as $\varphi=2 \pi x / \Lambda+m \varphi$ in the $x$ direction, where $\Lambda$ is the period, $m$ is the topological charge, and $\varphi$ is the azimuthal angle. Here, the $2 \times 2$ DVG is directly integrated by two orthogonal DVGs $\left(m_{x}=1\right.$ and $m_{y}=3$, normalized phase transition point: $x_{1}=y_{1}=0.5$ [20]). As shown in Figure 1a, the black regions represent 0 , while the white regions represent $\pi$. Such a phase pattern can be modulated via a geometric phase by in-plane controlling the optical axis orientation $\alpha$ :

$$
\alpha=\frac{\varphi_{\mathrm{DVG}}(x, y)}{2}+\alpha_{0}
$$

where $\varphi_{\mathrm{DVG}}(x, y)$ is the phase function of the DVG and $\alpha_{0}$ is the initial angle and usually assumed to be zero. The transmission formula of the $2 \times 2$ DVG can be expressed as:

$$
\mathrm{T}(x, y)=\exp \left(i \varphi_{\mathrm{DVG}}(x, y)\right)=\sum_{n_{x}=-\infty}^{+\infty} \sum_{n_{y}=-\infty}^{+\infty} C_{n_{x}, n_{y}} \exp \left[i\left(m_{x} n_{x}+m_{y} n_{y}\right) \varphi+\left(n_{x} x+n_{y} y\right) \frac{i 2 \pi}{\Lambda}\right],
$$

where $C_{n}$ is the coefficient of the $n^{\text {th }}$ diffraction order [21]. As $m_{x}=1$ and $m_{y}=3$, the incident beam will be mainly divided into 4 orders that carry the topological charge $m=n_{x}+3 n_{y}$. Special algorithms are used to calculate the number of phase transition points [20]. Here, for $x_{1}=y_{1}=0.5$, only simple $2 \mathrm{D}$ fork gratings that can generate a rectangular $2 \times 2$ optical vortex array are demonstrated. A more complicated matrix can be rationally designed by separately changing the number and values of the phase transition points. 


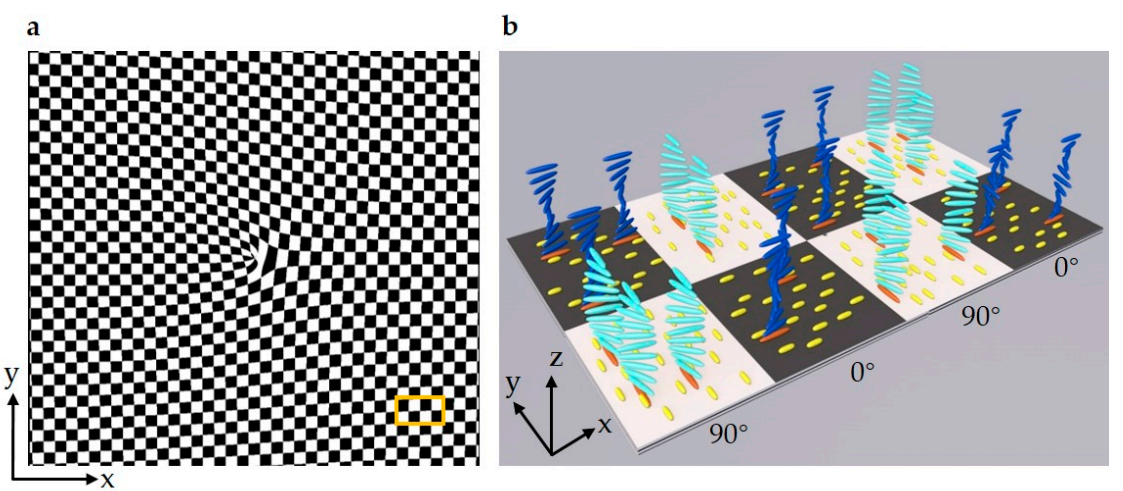

Figure 1. (a) The binary phase pattern of a $2 \times 2$ Damman vortex grating (DVG) with $m_{x}=1$ and $m_{y}=3$. (b) A schematic of liquid crystal (LC) directors in the yellow rectangle region labeled in (a).

The transmittance of the DVG can be analyzed by a Jones matrix and expressed as:

$$
\mathbf{T}=\mathbf{R}(-\alpha)\left[\begin{array}{cc}
\exp (-i \Gamma / 2) & 0 \\
0 & \exp (i \Gamma / 2)
\end{array}\right] \mathbf{R}(\alpha)=\cos \left(\frac{\Gamma}{2}\right) \mathbf{I}-i \sin \left(\frac{\Gamma}{2}\right)\left[\begin{array}{cc}
\cos 2 \alpha & \sin 2 \alpha \\
\sin 2 \alpha & -\cos 2 \alpha
\end{array}\right],
$$

where $\mathbf{R}$ is the rotation matrix, $\mathbf{I}$ is the identity matrix, $\Gamma=2 \pi \Delta n d / \lambda$ is the phase retardation, $\Delta n$ is the birefringence coefficient, $d$ indicates the thickness of the birefringent layer, and $\lambda$ is the free-space wavelength of incident light. Considering a circular polarized incidence, it can be expressed as $\mathrm{E}_{\mathrm{in}}=\frac{1}{\sqrt{2}}\left(\begin{array}{c}1 \\ \pm i\end{array}\right)$. Thus, the output can be depicted as:

$$
\mathbf{E}_{\text {out }}=\mathbf{T} \cdot \mathbf{E}_{\text {in }}=\frac{1}{\sqrt{2}} \cos \left(\frac{\Gamma}{2}\right)\left(\begin{array}{c}
1 \\
\pm i
\end{array}\right)-\frac{1}{\sqrt{2}} i \sin \left(\frac{\Gamma}{2}\right) \mathrm{e}^{ \pm i 2 \alpha}\left(\begin{array}{c}
1 \\
\mp i
\end{array}\right)
$$

when the halfwave condition satisfied, the incident left-circularly polarized light is handedness-converted and modulated with the designed phase. Meanwhile, for right-circularly polarized light, the opposite phase modulation is carried out.

The above design could be easily realized in an LC system where the optical axis orientation could be directly encoded with azimuthal angle control of LC directors [22]. As shown in Figure 1b, photoalignment agents indicated in yellow are utilized to perform the alignment, and the adjacent LC directors marked in orange follow the local guidance and further spread this order to the bulky LC. It needs to be noticed that here a double-layer reverse-twist configuration was adopted. Along the $z$ axis, the LC director rotates by a certain angle and then rotates back in the second layer. Adjacent LC domains with orthogonal planar alignment are represented by blue and cyan. These colors mean the LC helixes whose initial orientations are $0^{\circ}$ (blue) and $90^{\circ}$ (cyan), respectively, with respect to the $x$ axis. Here, instead of discrete wavelength compensation with birefringent phase retarders [23], the same starting and ending polarization with wavelength-dependent, different polarization revolution processes are introduced to overcome the dispersion [24,25].

A DVG is suitable for generating a series of OVs with different topological charges, while a Damman q-plate (DQP) [12] can produce an OV array with a same topological charge. Here, a hexagonal 2D Damman grating that consists of three 1D Damman gratings with relative angles of $60^{\circ}$ is encoded to a q-plate with $m=1$. Figure 2a illustrates the optical axis distributions of this DQP, where the color variation from black to white indicates the directions of the optical axis varying from $0^{\circ}$ to $180^{\circ}$. Figure $2 \mathrm{~b}$ shows the $3 \mathrm{D}$ scheme of the double-layer reverse-twist configuration in the center of the hexagonal DQP. When a circularly polarized incident light passes through this DQP, a hexagonal lattice array of OVs with orthogonal, circularly polarized states will be generated and will carry a topological charge of 1. 


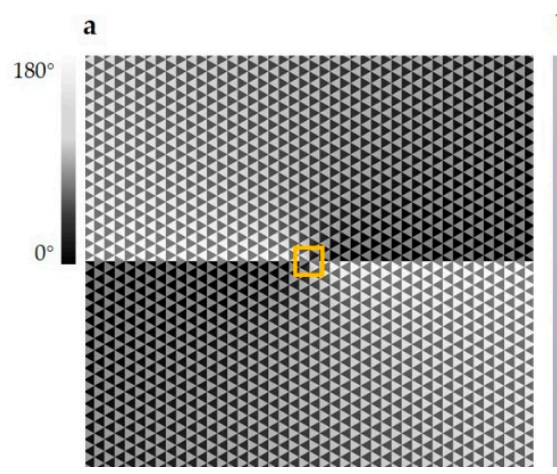

b

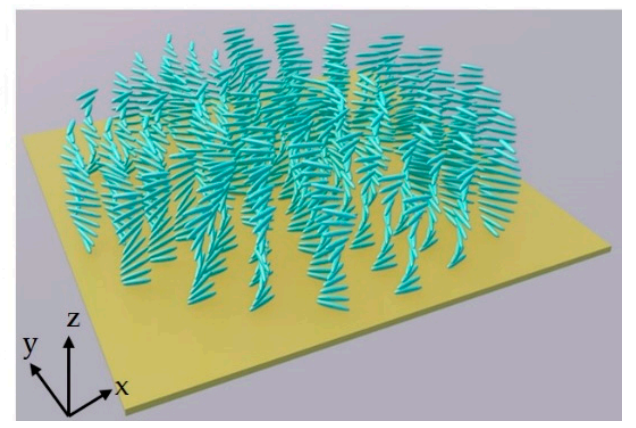

Figure 2. (a) The optical axis distributions of a hexagonal lattice Damman q-plate (DQP) with $m=1$.

(b) A schematic of LC directors in the yellow square region labeled in (a).

\subsection{Sample Preparation}

The indium-tin-oxide glass substrates were ultrasonically bathed and spin-coated with photoalignment agent SD1 (a polarization-sensitive sulfonic azo dye that was dissolved in dimethylformamide at a concentration of $0.3 \mathrm{wt} . \%$ ) [26,27]. After curing at $100{ }^{\circ} \mathrm{C}$ for $10 \mathrm{~min}$, the substrate was placed at the image plane of the Digital Micro-mirror Device (DMD)-based microlithography system $[28,29]$ to accomplish a uniform alignment, and then the DVG pattern was recorded with the orthogonal polarization exposure step. For the DQP, a 36-step partly overlapping exposure method was performed to carry out the director distributions.

The LCP [30] precursors (UCL017, Dai-Nippon Ink and Chemicals, Tokyo, Japan, dissolved in methylbenzene at a concentration of $23 \mathrm{wt} . \%$ ) mixed with right and left chiral dopants (R811 and S811 at a concentration of $1.27 \%$, Jiangsu Hecheng Display Technology, Nanjing, China), respectively, were spin-coated onto the SD1 membrane, layer by layer, at a speed of $600 \mathrm{r} / \mathrm{min}$, followed by a two minutes of UV exposure for each layer. The precursor with opposite chirality was spin coated at a speed of $1600 \mathrm{r} / \mathrm{min}$ to precisely control the double, right and left chiral dopant layers' thicknesses to be 1.59 and $1.76 \mu \mathrm{m}$, respectively. At this condition, the initial orientation of the LCP layer was kept the same to that of the termination one. The total LCP satisfied the halfwave condition, thus forming a broadband OV array generator.

\section{Results and Discussion}

Furthermore, in order to verify the broadband property, the planar DVG satisfied the $532 \mathrm{~nm}$ halfwave condition was fabricated and analyzed, in comparison to that of the double-layer reverse-twist configuration that could not. By adjusting the left-circularly polarized light incidents on the DVG, Figure $3 \mathrm{a}, \mathrm{g}$ is able to show the micrographs of the double-layer reverse-twist and planar $2 \times 2$ DVGs under crossed polarizers. Due to the discontinuity of LC directors at the boundary between neighboring orthogonal domains, dark lines, whose patterns are consisted with the designed phase patterns, can be observed. Figure $3 \mathrm{~b}-\mathrm{f}, \mathrm{h}-\mathrm{l}$ shows the diffraction patterns of the DVGs captured by a charge-coupled device (VTSE3S-2000, Vihent, Shanghai, China), with incident wavelengths of 473, 532, 700, 800, and $950 \mathrm{~nm}$, respectively. Due to the geometric phase modulation, the beam was diffracted to different orders carrying designed topological charges, e.g., as indicated by white circles in Figure 3b. As expected, donut-like OVs were obtained in different diffraction orders, and those with larger $m$ values exhibited larger phase singularities and ring radii [31]. Figure $3 b-f$ indicates nearly constant diffraction efficiency across the range of 530-930 $\mathrm{nm}$. Meanwhile, for the planar DVG, the diffraction efficiency reached a maximum at $532 \mathrm{~nm}$, in which the halfwave condition was perfectly satisfied. The efficiency decreased significantly when the wavelength deviated from $532 \mathrm{~nm}$ (Figure 3h-1). Additionally, thanks to the dependency of diffraction angle on incident wavelengths, the pattern became larger along with the increasing wavelength. A comparison between above two samples verified both the faithful phase encoding and the equally high broadband efficiency of the double-layer reverse-twist DVGs. 


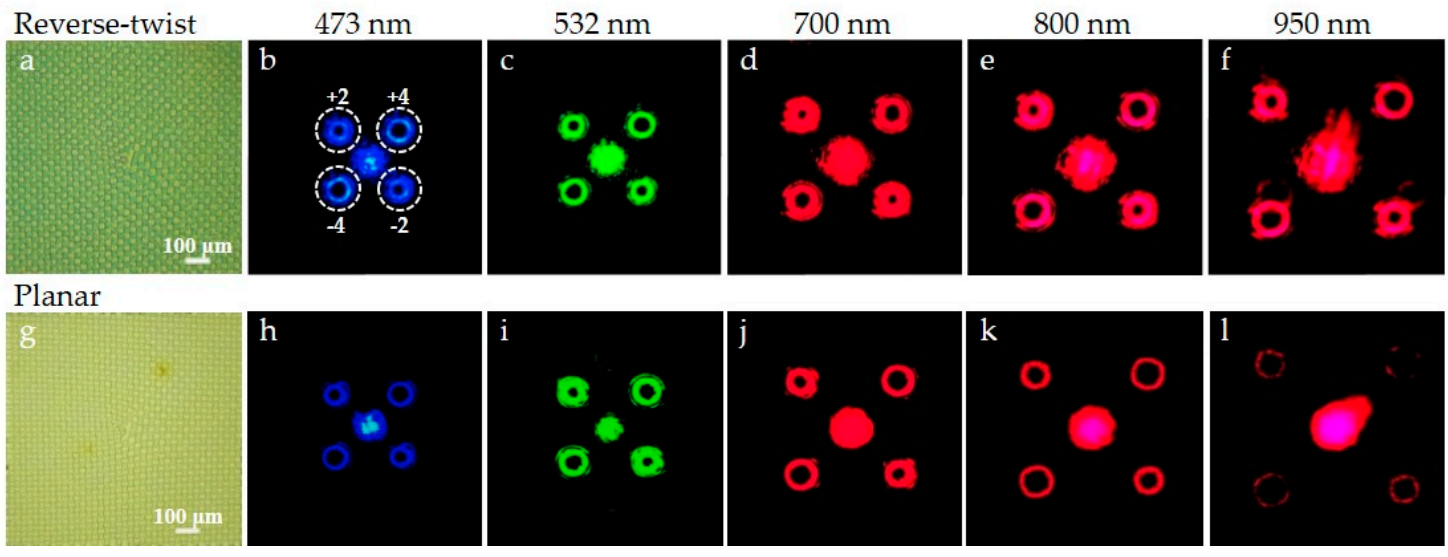

Figure 3. The micrographs of (a) double-layer reverse-twist and (g) planar $2 \times 2$ DVGs under crossed polarizers; (b-f) and (h-1) the corresponding diffraction patterns at 473, 532, 700, 800, and $950 \mathrm{~nm}$, respectively.

Double-layer reverse-twist and planar DQPs were also demonstrated. The incident was also left-circularly polarized light. The samples revealed hexagonal lattice patterns consistent with the designed optical axis distributions under crossed polarizers (Figure 4a,g). The continuous color or gray scale variations in the micrographs were due to the azimuthal angle changes of the LC directors. The diffraction patterns presented in Figure $4 \mathrm{~b}-\mathrm{f}, \mathrm{h}-\mathrm{l}$ clearly show a regular hexagonal lattice of diffraction orders with the same $m$ of 1 . By presetting the lattice symmetry, the pitch of the Damman grating, and the topological charge of the q-plate, the OV arrays could be rationally manipulated. An obviously different dependency on wavelength between the two different designs was also verified.
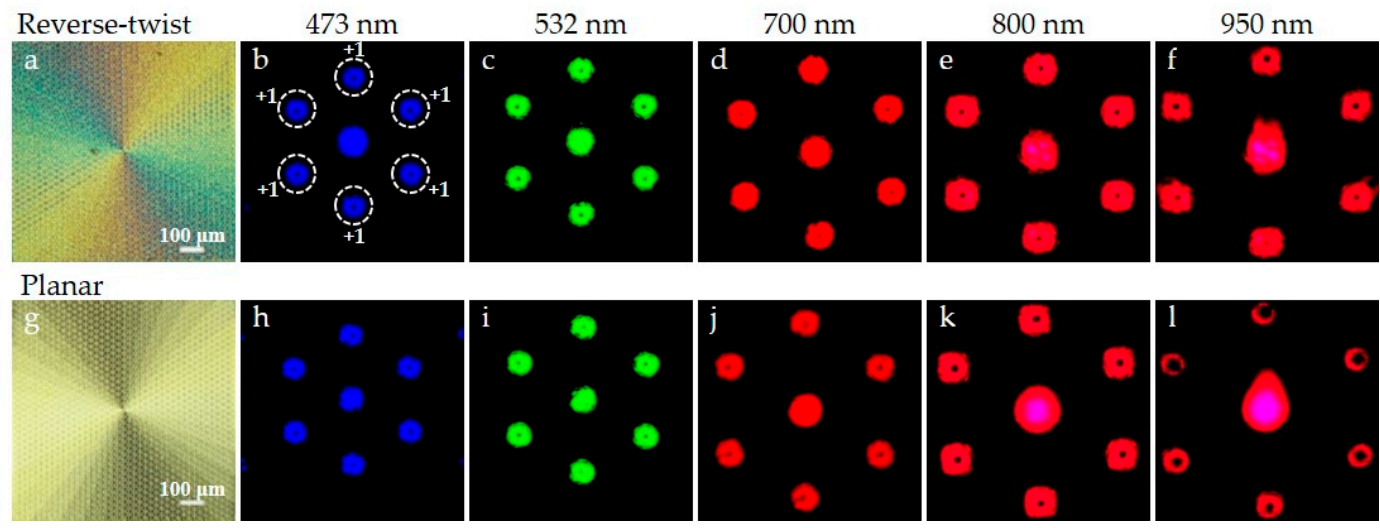

Figure 4. The micrographs of (a) double-layer reverse-twist and (g) planar hexagonal lattice DQPs under crossed polarizers; (b-f) and (h-l) the corresponding diffraction patterns at 473, 532, 700, 800, and $950 \mathrm{~nm}$, respectively.

Moreover, diffraction efficiencies at several wavelengths were quantitively detected and are depicted in Figure 5. The efficiency was defined as the intensities of total first orders divided by all transmitted energy. Theoretically, it was $65.6 \%$ for $2 \times 2$ DVG [20]. The measured data for the double-layer reverse-twist and planar DVGs are depicted by red and black dots in Figure 5 a, respectively. The red dots varied in the range of $40.1-49.7 \%$ for the bandwidth of $530-930 \mathrm{~nm}$, which was comparable to the theoretical value in a broadband. The diffraction efficiency of the planar DVG exhibited a maximum of $42.7 \%$ at $532 \mathrm{~nm}$, and it decreased gradually along with the wavelength away from $532 \mathrm{~nm}$, i.e., the halfwave condition. Figure $5 \mathrm{~b}$ reveals the diffraction efficiencies of double-layer reverse-twist and planar DQPs. A quite similar phenomenon was observed. The red curve kept over $51.0 \%$ in the range of $530-930 \mathrm{~nm}$, while the maximum efficiency of $57.2 \%$ was reached at $532 \mathrm{~nm}$ for the planar DQP (black), and the efficiency decreased rapidly along with the wavelength away from 
$532 \mathrm{~nm}$. The curves matched well with the intensity variation of the OV arrays in Figures 3 and 4. The results vividly verified the broadband OV array generations.
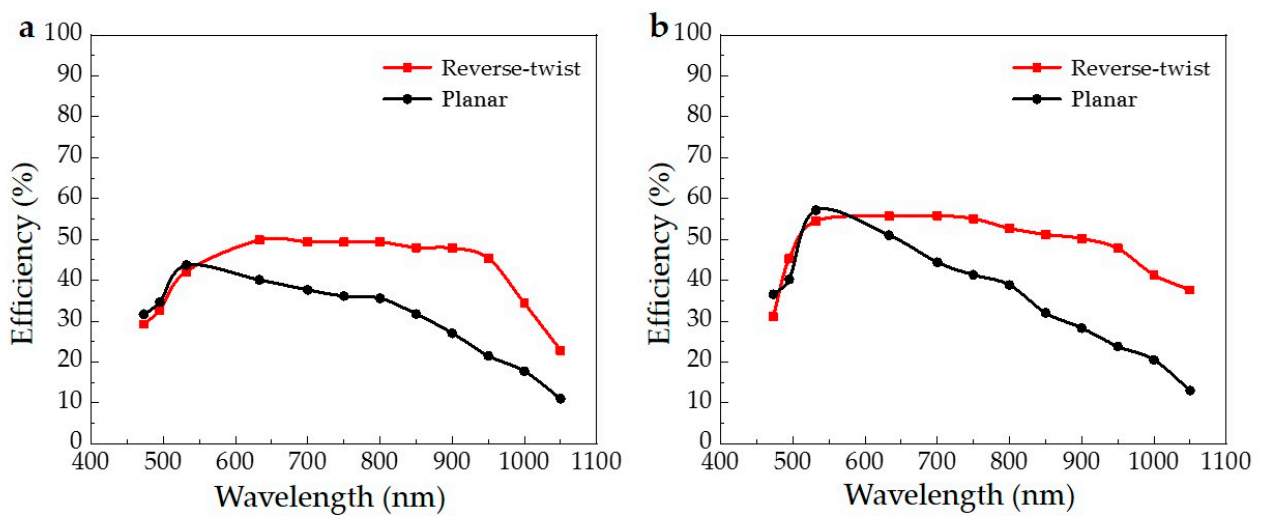

Figure 5. The dependencies of diffraction efficiencies on wavelength. (a) DVGs and (b) DQPs.

\section{Conclusions}

A double-layer reverse-twist configuration was introduced into the planar geometric phase optics elements to realize broadband multichannel OV generators. By encoding DVG and DQP phase profiles to photopatterned LCP layers, multichannel rectangular and hexagonal OV arrays were generated equally-efficiently with mode conversion efficiencies exceeding $40.1 \%$ and $51.0 \%$, respectively, across a broadband wavelength range over $400 \mathrm{~nm}$. These devices showed significant superiorities in the working band compared to the planar ones whose efficiency maximized at the halfwave condition and dropped when deviating from the designed wavelength. Thanks to the fidelity and flexibility of photoalignment technology, arbitrary phase patterns could be encoded to the double-layer reverse-twist LCP systems. This supplies an easy but efficient fabrication method for broadband beam shaping elements, thus paving a bright way for advanced photonic applications such as wavelength-division multiplexing-compatible mode encoding, high-density information storage, and encryption.

Author Contributions: W.D. and W.H. conceived the original idea; H.Z., W.D., T.W., and C.X. performed the experiments, analyzed the data and wrote the manuscript; W.H. supervised and directed the research. All authors have read and agreed to the published version of the manuscript.

Funding: This work was supported by the National Natural Science Foundation of China (No. 61922038) and the Fundamental Research Funds for the Central Universities (14380170) and the Tang Scholar program.

Conflicts of Interest: The authors declare no conflict of interest.

\section{References}

1. Coullet, P.; Gil, L.; Rocca, F. Optical vortices. Opt. Commun. 1989, 73, 403-408.

2. Allen, L.; Beijersbergen, M.W.; Spreeuw, R.J.; Woerdman, J.P. Orbital angular momentum of light and the transformation of laguerre-Gaussian laser modes. Phys. Rev. A 1992, 45, 8185-8189. [PubMed]

3. Marrucci, L.; Manzo, C.; Paparo, D. Optical spin-to-orbital angular momentum conversion in inhomogeneous anisotropic media. Phys. Rev. Lett. 2006, 96, 163905. [PubMed]

4. Gecevicius, M.; Drevinskas, R.; Beresna, M.; Kazansky, P.G. Single beam optical vortex tweezers with tunable orbital angular momentum. Appl. Phys. Lett. 2014, 104, 288-299.

5. D’Ambrosio, V.; Spagnolo, N.; Re, L.D.; Slussarenko, S.; Li, Y.; Kwek, L.C.; Sciarrino, F. Photonic polarization gears for ultra-sensitive angular measurements. Nat. Commun. 2013, 4, 2432.

6. Cardano, F.; Massa, F.; Qassim, H.; Karimi, E.; Slussarenko, S.; Paparo, D.; Marrucci, L. Quantum walks and wavepacket dynamics on a lattice with twisted photons. Sci. Adv. 2015, 1, e1500087.

7. Kumar, A.; Prabhakar, S.; Vaity, P.; Singh, R.P. Information content of optical vortex fields. Opt. Lett. 2011, 36, 1161-1163. 
8. Wang, Z.; Zhang, N.; Yuan, X.C. High-volume optical vortex multiplexing and de-multiplexing for free-space optical communication. Opt. Express 2011, 19, 482-492.

9. Tamburini, F.; Mari, E.; Sponselli, A.; Thid, B.; Bianchini, A.; Romanato, F. Encoding many channels on the same frequency through radio vorticity: First experimental test. New J. Phys. 2012, 14, 033001.

10. Awaji, Y.; Wada, N.; Toda, Y. Demonstration of spatial mode division multiplexing using Laguerre-Gaussian mode beam in telecom-wavelength. In Proceedings of the 2010 IEEE Photonics Society's 23rd Annual Meeting, Denver, CO, USA, 7-11 November 2010.

11. Gibson, G.; Courtial, J.; Padgett, M.J.; Vasnetsov, M.; Franke-Arnold, S. Free-space information transfer using light beams carrying orbital angular momentum. Opt. Express 2004, 12, 5448-5456.

12. Chen, P.; Ge, S.J.; Duan, W.; Wei, B.Y.; Cui, G.X.; Hu, W.; Lu, Y.Q. Digitalized Geometric Phases for Parallel Optical Spin and Orbital Angular Momentum Encoding. ACS Photonics 2017, 4, 1333-1338.

13. Kobashi, J.; Yoshida, H.; Ozaki, M. Planar optics with patterned chiral liquid crystals. Nat. Photonics 2016, 10, 389-392.

14. Rafayelyan, M.S.; Tkachenko, G.; Brasselet, E. Reflective Spin-Orbit Geometric Phase from Chiral Anisotropic Optical Media. Phys. Rev. Lett. 2016, 116, 253902. [PubMed]

15. Kobashi, J.; Yoshida, H.; Ozaki, M. Polychromatic Optical Vortex Generation from Patterned Cholesteric Liquid Crystals. Phys. Rev. Lett. 2016, 116, 253903.

16. Barboza, R.; Bortolozzo, U.; Clerc, M.G.; Residori, S. Berry Phase of Light under Bragg Reflection by Chiral Liquid-Crystal Media. Phys. Rev. Lett. 2016, 117, 053903.

17. Chen, P.; Ma, L.L.; Duan, W.; Chen, J.; Ge, S.J.; Zhu, Z.H.; Lu, Y.Q. Digitalizing Self-Assembled Chiral Superstructures for Optical Vortex Processing. Adv. Mater. 2018, 30, 1705865.

18. Dammann, H.; Klotz, E. Coherent Optical Generation and Inspection of Two-dimensional Periodic Structures. J. Mod. Opt. 1977, 24, 505-515.

19. Fan, F.; Yao, L.; Wang, X.; Shi, L.; Srivastava, A.K.; Chigrinov, V.G.; Kwok, H.-S.; Wen, S. Ferroelectric Liquid Crystal Dammann Grating by Patterned Photoalignment. Crystals 2017, 7, 79.

20. Zhou, C.; Liu, L. Numerical study of Dammann array illuminators. Appl. Opt. 1995, 34, 5961-5969.

21. Yu, J.; Zhou, C.; Jia, W.; Hu, A.; Cao, W.; Wu, J.; Wang, S. Three-dimensional Dammann vortex array with tunable topological charge. Appl. Opt. 2012, 51, 2485-2490.

22. Chen, P.; Wei, B.Y.; Hu, W.; Lu, Y.Q. Liquid-Crystal-Mediated Geometric Phase: From Transmissive to natBroadband Reflective Planar Optics. Adv. Mater. 2019, 1903665. [CrossRef]

23. Samoylov, A.V.; Samoylov, V.S.; Vidmachenko, A.P.; Perekhod, A.V. Achromatic and super-achromatic zero-order waveplates. J. Quant. Spectrosc. Radiat. Transf. 2004, 88, 319-325.

24. Komanduri, R.K.; Lawler, K.F.; Escuti, M.J. Multi-twist retarders: Broadband retardation control using self-aligning reactive liquid crystal layers. Opt. Express 2013, 21, 404-420. [PubMed]

25. Tang, S.T.; Kwok, H.S. Mueller calculus and perfect polarization conversion modes in liquid crystal displays. J. Appl. Phys. 2001, 89, 5288-5294.

26. Chigrinov, V.G.; Pikin, S.A.; Verevochnikov, A.M.; Kozenkov, V.M.; Khazimullin, M.V.; Ho, J.Y.; Kwok, H.S. Diffusion model of photoaligning in azo-dye layers. Phys. Rev. E 2004, 69, 061713.

27. Chigrinov, V.G.; Prudnikova, E.; Kozenkov, V.M.; Kwok, H.S.; Akiyama, H.; Kawara, T.; Takatsu, H. Synthesis and properties of azo dye aligning layers for liquid crystal cells. Liq. Cryst. 2002, 29, 1321-1327.

28. Wu, H.; Hu, W.; Hu, H.C.; Lin, X.W.; Zhu, G.; Choi, J.W. Arbitrary photo-patterning in liquid crystal alignments using DMD based lithography system. Opt. Express 2012, 20, 16684-16689.

29. Duan, W.; Chen, P.; Ge, S.J.; Liang, X.; Hu, W. A Fast-Response and Helicity-Dependent Lens Enabled by Micro-Patterned Dual-Frequency Liquid Crystals. Crystals 2019, 9, 111.

30. White, T.J.; Broer, D.J. Programmable and adaptive mechanics with liquid crystal polymer networks and elastomers. Nat. Mater. 2015, 14, 1087-1098.

31. Yao, A.M.; Padgett, M.J. Orbital angular momentum: Origins, behavior and applications. Adv. Opt. Photonics 2011, 3, 161-204.

(C) 2020 by the authors. Licensee MDPI, Basel, Switzerland. This article is an open access article distributed under the terms and conditions of the Creative Commons Attribution (CC BY) license (http://creativecommons.org/licenses/by/4.0/). 